$\begin{array}{ll}\text { Research Square } & \begin{array}{l}\text { Preprints are preliminary reports that have not undergone peer review. } \\ \text { They should not be considered conclusive, used to inform clinical practice, } \\ \text { or referenced by the media as validated information. }\end{array}\end{array}$

\title{
Ascorbic acid and Proline alleviat the adverse effects of salinity stress in Duranta erecta L. var. Variegata plants
}

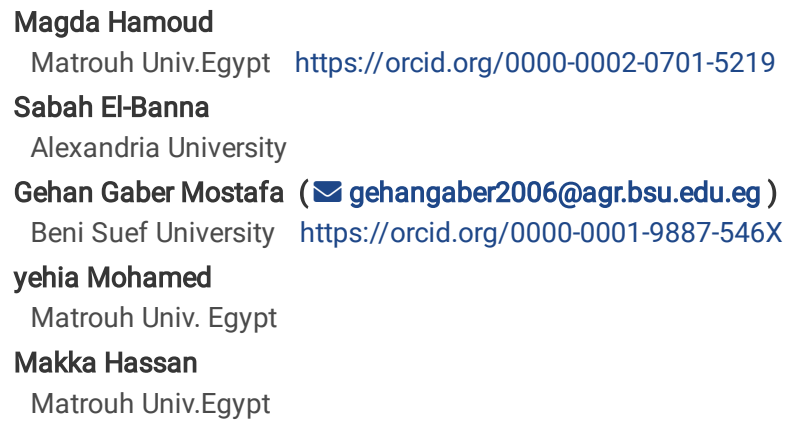

Research Article

Keywords: salinity stress, antioxidant enzymes, DNA breakage, Duranta, proline, ascorbic acid

Posted Date: February 17th, 2022

DOI: https://doi.org/10.21203/rs.3.rs-1343974/v1

License: (c) (7) This work is licensed under a Creative Commons Attribution 4.0 International License. Read Full License 


\section{Abstract}

The present study was carried out at the Faculty of Desert Agriculture and Environmental in Fouka, Matrouh University (Matrouh governorate) Egypt, during the two successive seasons of 2019/2020 and 2020/2021. This investigatin aimed to study the effects of ascorbic acid (0.0, 100, 200 ppm) and proline (0.0, 40, $80 \mathrm{ppm}$ ) on alleviating the adverse effects of salinity stress at 0.0,2000, 4000, $6000 \mathrm{ppm}$ seawater in Duranta erecta L. var. Variegata plants. The experiment was designed in a split-plot design in random completely block design (RCBD) with three replications. In this experiment, the main plot was salt water levels and the sub-plot was spraying by proline and ascorbic acid treatments. The results showed that treated plants with proline at 80 ppm and ascorbic acid at 200ppm enhanced plant height, leaves dry weight, leaf green color degree (SPAD units), plant survival percentage, stomata measurement, potassium of leaves and antioxidant such as superoxide dismutase (SOD), catalase (CAT), reduced glutathione (GSH), cytochrome b5, cytochrome P450 (CYP450), amidopyrine Ndemethylase and aniline 4-hydroxylase activity. Moreover, treatments deacreased thiobarbituric acid reactive substances (TBARS), sodium and chlorid. Finaly, proline and ascorbic acid alleviate the negative effects of salinity and increase Duranta plant growth and plant survival percentage by inhancing the antioxidant enzymes, potassium cations and photosynthetic system, decresing DNA breakage \%, harmful ions eg:sodium and chloride.

\section{Introduction}

Duranta erecta L. var. Variegata, belongs to the family Verbenaceae, is a small tree or a vigorous shrub and a sprawling shrub. It is planted frequently for the beauty of white and green leaves and blue tubular flowers appearances almost all year round (Huxley et al. 1992). It is grow in tropical and subtropical areas and used for accent plants (Pipattanawong et al. 2008).

Salinity reduces plant growth, exposure of plants for long periods to salinity or severe salinity leads to death of plants. Salinity is a major concern in arid and semi-arid regions, especially the region that have not enough amount of rain to wash the soil from salts, and propten exacerbated by climate change (Ramawat 2010). Salinity also modifies gene expression (Soussi et al. 2001) increased the levels of reactive oxygen species (ROS) (Apel and Hirt 2004), chlorophyll breakdown (Kato and Shimizu 1987) and many other effects such as leaf senescence (Allu et al. 2014). Gene expression leads to the emergence of new proteins that are not present before the stimulation process. It is known that in recent time's fresh water resources have been greatly depleted, so it is necessary to try to use different alternatives sources, such as desalinated water or ground water that suffers from salinity. Salinity problems have become more prevalent in the past ten years, using of alternative non-potable water become a major contributor to irrigation (Marcum 2006). From the above, it is important to choose tolerant plant varieties and species or search about some subatances can help plants to tolerat ahigh levels of salinity (Hassanain et al. 2017). Several enzymes help detoxify reactive oxygen species. Superoxide dismutase is the first defense enzyme that converts superoxide into H2O2 (Sairam et al. 2005).

Ascorbic acid $\left(\mathrm{C}_{6} \mathrm{H}_{8} \mathrm{O}_{6}\right)$ is a small molecular soluble in water and insoluble in other solvents as chloroform, benzene and fats. Ascorbic acid acts as an antioxidant, working to remove enzymatic toxins such as $\mathrm{H}_{2} \mathrm{O}_{2}$ and other harmful factors that affect plant photosynthesis (Galal et al. 2000). It is a reductant and reacting with and/or scavenging many types of free radicals. Ascorbic acid reacts non-enzymatically with superoxide, hydrogen peroxide and singlet oxygen (Pourcel et al. 2007). Proline is an amino acid that preserves the plant cell under conditions of drought and salinity where it prevents and reduces the breakdown of protein in the cell and maintains the vitality of the plant. Proline acts as a visceral osmotic solute and as a source of energy and nitrogen, so proline has a role in reducing the harmful effects of salt stress (Venkatesan and Chellappan 1998).

This investigation aimed to study, the effects of ascorbic acid and proline on alleviating the adverse effects of salinity stress in Duranta erecta L. var. Variegata plants.

\section{Material And Methods}

The present study was carried out during two successive seasons of (2019-2020) and (2020-2021) at the Faculty of Desert Agriculture and Environmental in Fouka, Matrouh University (Matrouh governorate) Egypt, to investigate the effects of proline and ascorbic acid on the growth, some chemical and biochemical composition of Duranta erecta L Var. Variegata plants under salinity stress condition. One year old plants were obtained from commercial (private) nursery for ornamental plants in Marsa Matrouh city. The plants had a uniform sizes and shapes, with 5 branches / plant and $70 \mathrm{~cm}$ in height were used. One plant / pot was cultivated. The used soil is a mixture of $50 \%$ sandy soil (the available soil in the farm of Faculty of Desert Agriculture and Environmental in Fouka, Matrouh University and $50 \%$ clay soil. Physical and chemical soil analysis is shown in Table (1).

Treatments and cultural aspects: four concentrations of the seawater irrigation (tap water, 2000, 4000 and 6000 ppm), and two anti - salt stress materials: proline at 40 and 80 ppm and ascorbic acid at 100 and 200 ppm were used. Plants were sprayed with anti-salt stress materials and spraying with tap water as a control until run off points. Duranta plants were planted in pots of $30 \mathrm{~cm}$ diameter on 20 th of December 2019 in the first season and 20 th of December 2020 in the second season. Plants sprayed directly with anti-stress materials repeated every two weeks for 4 months. Irrigation was carried out twice a week with saline water and irrigated with tap water once every two weeks. Plants were irrigated three times a week from May to the end of the experiment (20 August in both seasons) with saline water and irrigation with tap water once every two weeks until 20th of August. On the other hand, spraying plants with anti-salt stress compounds was stoped after the first cut (four months fom the beginning of the experiment) while irrigation with seawater concentration was continued to the end of the experiment. 
Table 1

Physical and chemical analysis of the soil

\begin{tabular}{|ll|}
\hline Physical properties & Chemical properties \\
\hline Clay: $21.78 \%$ & $\mathrm{pH} 8.34$ \\
Silt: $11.54 \%$ & $\mathrm{E} . \mathrm{C}(\mathrm{ds} / \mathrm{m}) 8.20$ \\
Soil type: Sandy clay loam. & $\mathrm{O}$.M $\% 1.5$ \\
& $\mathrm{CaCo}_{3} \% 24.53$ \\
& $\mathrm{Hco}_{3}^{-}\left(\mathrm{meq} \mathrm{L}^{-1}\right) 4.7$ \\
& $\mathrm{Cl}^{-}\left(\mathrm{meq} \mathrm{L}^{-1}\right) 90.1$ \\
& $\mathrm{SO}^{-}\left(\mathrm{meq} \mathrm{L}^{-1}\right) 38.5$ \\
& $\mathrm{Ca}^{++}\left(\mathrm{meq} \mathrm{L}^{-1}\right) 36.8$ \\
& $\mathrm{Na}^{+}\left(\mathrm{meq} \mathrm{L}^{-1}\right) 65.2$ \\
& $\mathrm{Mg}^{++}\left(\mathrm{meq} \mathrm{L}^{-1}\right) 28.4$ \\
& $\mathrm{~K}^{+}\left(\mathrm{meq} \mathrm{L}^{-1}\right) 2.9$ \\
\hline
\end{tabular}

\section{Recorded data}

data was collected twice per season, after four and eight months for the first and second cut respectively.

Plant height $(\mathrm{cm})$ was measured.

Leaves dry weight per branch $(\mathrm{g})$ leaves dry weight was estimated after dried on oven at $70^{\circ} \mathrm{C}$ for 72 hours.

Plant survival was estimated as the percentage of plants survival (how many plants kept growing to that failed).

\section{Leaf green color degree (SPAD units)}

It was determined on the fresh leaves as indicator to chlorophyll content. The determination was done according to the method described by Yadawa (1986) using Minolta SPAD Chlorophyll Meter model-502.

\section{Stomata measurements}

Number, length and diameter of stomata were calculated as the methods of Dore (1986). Individual leaves (4th leaf) were first removed from stem. Leaf a baxial epidermis cleaned using a degreased cotton ball and then carefully smeared with nail varnish in the mid-area between the central vein and leaf edge for approximately $20 \mathrm{~min}$. Thin film was peeled off from the leaf surface, placed into a microscope slide after one drop of tap water was added then the cover glass closed. Under a light microscope, number of stomata was counted, as well as length and diameter of three stomata per leaf were measured magnifying by $100 \times 10$ (objective $\times$ oculars). Values obtained were multiplied by 1.25 ; a coefficient obtained by adjusting the ocular micrometer.

\section{Potassium contents of leaves (\%)}

Potassium percentage was determined photometrically in the acid digested samples by using flam photometer as described by Page et al. (1982).

\section{Sodium contents of leaves (\%)}

Sodium percentage was determined according to Piper. (1967).

Chloride contents of leaves (\%)

Chloride percentage was determined according to Piper. (1967).

\section{Determination of antioxidant enzymes: Enzyme extraction}

Leaves were taken to estimate the enzymes. Once after 120 days and once again after 240 days from treatment. Sample preparation was done as described by Mukherjee and Choudhuri (1983). A fresh sample $(250 \mathrm{mg})$ was frozen in liquid nitrogen and finely ground by pestle in a chilled motor, the frozen powder was added to $10 \mathrm{ml}$ of $100 \mathrm{mM}$ phosphate buffer $\left(\mathrm{KH}_{2} \mathrm{PO}_{4} / \mathrm{K}_{2} \mathrm{HPO}_{4}, \mathrm{pH} 7.0\right)$ containing $0.1 \mathrm{mM} \mathrm{Na} 2 \mathrm{EDTA}$ and $0.1 \mathrm{~g}$ of polyvinylpyrrolidone (PVP), the homogenate was filtered through cheese cloth then centrifuged at $15000 \mathrm{~g}$ for $10 \mathrm{~min}$. The supernatant was recentrifuged at $18000 \mathrm{~g}$ for $10 \mathrm{~min}$; the resulted supernatant was collected and stored at $4{ }^{\circ} \mathrm{C}$ for assay of superoxide dismutase (SOD), catalase (CAT), thiobarbituric acid reactive substances (TBARS), reduced glutathione (GSH), cytochrome b5, cytochrome P450 (CYP450), amidopyrine N-demethylase and aniline 4-hydroxylase activity. 
Thiobarbituric acid reactive substances(TBARS)were measured as described by Tappel and Zalkin (1959).

Reduced glutathione(GSH) was determined according to the method described by Ellman (1959).

Catalase (CAT; EC 1.11.1.6) was determined according to the method described by Sinha (1972).

Superoxide dismutase (SOD, EC 1.15.1.1) was assayed in plant tissue extracts according to the method described by Nishikimiet al. (1972).

Determination of oxdative stress and biomarkers

Assay of cytochrome b5 and cytochrome P450 were estimated by the method of Omura and Sato (1964).

The activity of amidopyrine N-demethylase was measured according to Nash (1953).

The activity of aniline 4-hydroxylase was measured according to Kato and Gillette (1965).

The diphenylamine DNA fragmentation percentage assay is a very useful method for measuring apoptosis by determining the percentage of fragmentation of DNA into oligosomal-sized fragments. Plants DNA breakage was conducted using the procedure of Wu et al. (2006).

\section{Experimental layout and statistical analysis}

The experiment was designed in a split-plot design in RCBD with three replications. In this experiment, salt water levels expressed as the main plot and the sub-plot was spraying by proline and ascorbic acid treatments. Each experiment was included 20 treatments, 4 levels of salt-water irrigation and 5 treatments of anti-stress compounds (control, 2 rates proline and 2 rates ascorbic acid). 4 pots for each treatment per replicate and each pot contained 1 plant). The collected data in this study were subjected to analysis of variance (ANOVA) using CoStat program. Least significant difference (LSD) was used at 0.05 level of probability to test differences between treatments (Gomez and Gomez. 1984).

\section{Results}

\section{Vegetative traits}

All salinity concentrations decreased significantly plant height and leaves dry weight per branch in both seasons and cuttings without using anti-stress compounds as shown in Table (2). Plant irrigation with 6000 ppm treatment gave the lowest values and died befor second cut. While, addition of proline and ascorbic acid at all concentrations inhanced plant growth and increased significantly plant height and leves dry weight in both seasons and cuttings. The concentrations of 80 ppm proline gave the best results it increased plant height by $8.0,18.0,9.4$ and $18.44 \%$ for the first and second cut of the first and second seasons respectively. In addition, it increased leaves dry weight per branch by $94.4,131.8,96.3$ and $131.3 \%$ for first and second cut of the first and second seasons respectively.

\section{Chemical composition}

Seawater irrigation at all levels of salinity dereased significantly total chlorophyll content (SPAD unit) and potassium of leaves \% in the absence of proine and ascorbic acid in the both cutting and seasons as shown in Table (3). Seawater at 6000 ppm gave the lowest vaues in the first cut of both seasons. On the other hand, all concentrations of ascorbic acid and proline increased significantly total chlorophyll content and potassium of leaves \% in both cuttings of both seasons. Spraying plants with proline at $80 \mathrm{ppm}$ gave the highest values followed by ascorbic acid at 200 ppm compared to untreated plants as the mean effect for total chlorophyll content. This increment valued by 23.4, 29.5, 26.92 and $12.9 \%$ in the first and second cut of the first and second season. For potassium of leaves \% the increment valued by $98.3,56.68,85.7$ and $107.1 \%$ for the first and second cut of the first and second seasons respectively compared with untreated plants.

As the contrary effects, all salinity levels increased significantly sodium and chlorid of leaves in both seasons and cuttings as shown in Table (4). The concentration of $6000 \mathrm{ppm}$ gave the highest values in the first cut of both seasons. 
Table 2

Effects of ascorbic acid and proline and their interaction treatments on plant height $(\mathrm{cm})$ and leaves dry weight (g) of Duranta erecta $L$ Var. Variegata plants under salinity stress condition at 2019/2020 and 2020/2021

\begin{tabular}{|c|c|c|c|c|c|c|c|c|c|c|c|c|}
\hline \multicolumn{7}{|l|}{$2019 / 2020$ season } & \multicolumn{6}{|c|}{$2020 / 2021$ season } \\
\hline \multirow{4}{*}{$\begin{array}{l}\text { Saline water level } \\
\text { (ppm) }\end{array}$} & \multicolumn{6}{|c|}{ Proline (P) and Ascorbic (As) concentration (ppm) } & \multicolumn{6}{|c|}{ Proline (P) and Ascorbic (As) concentration (ppm) } \\
\hline & 0.0 & As 100 & As200 & P40 & P80 & $\begin{array}{l}\text { Means } \\
\text { (S) }\end{array}$ & 0.0 & As 100 & As200 & P40 & P80 & $\begin{array}{l}\text { Means } \\
\text { (S) }\end{array}$ \\
\hline & \multicolumn{12}{|c|}{ Plant height } \\
\hline & \multicolumn{6}{|c|}{ First cut } & \multicolumn{6}{|c|}{ First cut } \\
\hline 0.0 & 93.03 & 96.10 & 92.37 & 96.10 & 93.00 & 92.37 & 93.73 & 88.03 & 93.07 & 96.10 & 96.67 & 93.52 \\
\hline 2000 & 83.57 & 87.10 & 92.43 & 94.03 & 94.33 & 90.29 & 83.37 & 87.10 & 92.93 & 94.07 & 95.00 & 90.49 \\
\hline 4000 & 84.60 & 96.53 & 90.57 & 93.37 & 92.23 & 91.46 & 84.90 & 97.23 & 89.63 & 91.93 & 91.67 & 91.07 \\
\hline 6000 & 73.33 & 78.03 & 73.23 & 80.3 & 81.87 & 77.35 & 71.60 & 77.73 & 73.30 & 79.78 & 81.70 & 76.82 \\
\hline Means & 83.63 & 87.25 & 87.15 & 90.95 & 90.36 & & 83.40 & 87.52 & 87.23 & 90.47 & 91.26 & \\
\hline \multirow[t]{2}{*}{ LSD at $5 \%$} & \multicolumn{6}{|c|}{$S=3.26^{* \star} F=3.51^{\star \star \star}(S) *(F)=7.02^{\star \star \star}$} & \multicolumn{6}{|c|}{$S=2.43^{\star \star \star} F=3.14^{\star \star \star}(S) *(F)=6.27^{\star}$} \\
\hline & \multicolumn{6}{|c|}{ Second cut } & \multicolumn{6}{|c|}{ Second cut } \\
\hline 0.0 & 117.1 & 100.87 & 107.23 & 123.33 & 124.5 & 114.61 & 116.83 & 102.23 & 108.90 & 123.77 & 124.83 & 115.31 \\
\hline 2000 & 89.83 & 99.47 & 106.30 & 110.77 & 122.57 & 105.78 & 89.87 & 99.07 & 105.57 & 113.47 & 122.57 & 106.11 \\
\hline 4000 & 100.9 & 106.6 & 109.50 & 107.37 & 116.20 & 108.11 & 100.30 & 106.27 & 109.33 & 108.07 & 116.20 & 108.03 \\
\hline Means & 102.61 & 102.31 & 107.68 & 113.82 & 121.09 & & 102.33 & 102.52 & 107.93 & 115.10 & 121.20 & \\
\hline \multirow[t]{3}{*}{ LSD at $5 \%$} & \multicolumn{6}{|c|}{$S=2.62^{\text {ns }} F=3.65^{*}(S) *(F)=2.06^{n s}$} & \multicolumn{6}{|c|}{$S=2.23^{\star * \star} F=3.68^{* * *}(S) *(F)=6.37^{* \star *}$} \\
\hline & \multicolumn{12}{|c|}{ Leaves dry weight per branch } \\
\hline & \multicolumn{6}{|c|}{ First cut } & \multicolumn{6}{|c|}{ First cut } \\
\hline 0.0 & 4.84 & 6.45 & 6.48 & 6.41 & 6.36 & 6.11 & 4.77 & 6.37 & 6.52 & 6.28 & 6.37 & 6.06 \\
\hline 2000 & 4.03 & 5.76 & 6.93 & 6.23 & 8.38 & 6.26 & 4.03 & 5.79 & 6.99 & 6.09 & 8.36 & 6.25 \\
\hline 4000 & 3.39 & 5.80 & 5.88 & 6.10 & 8.09 & 5.85 & 3.23 & 5.62 & 5.89 & 6.05 & 7.93 & 5.74 \\
\hline 6000 & 2.08 & 2.84 & 2.85 & 3.07 & 5.09 & 3.19 & 2.05 & 2.75 & 3.00 & 2.95 & 5.00 & 3.15 \\
\hline Means & 3.59 & 5.21 & 5.53 & 5.45 & 6.98 & & 3.52 & 5.13 & 5.60 & 5.34 & 6.91 & \\
\hline \multirow[t]{2}{*}{ LSD at $5 \%$} & \multicolumn{6}{|c|}{$S=0.79^{\star \star \star} F=0.64^{\star \star \star}(S) *(F)=1.29^{\star}$} & \multicolumn{6}{|c|}{$S=0.76^{\star \star \star} F=0.59^{\star \star \star}(S) *(F)=1.19^{\star}$} \\
\hline & \multicolumn{6}{|c|}{ Second cut } & \multicolumn{6}{|c|}{ Second cut } \\
\hline 0.0 & 5.28 & 6.98 & 7.76 & 6.67 & 10.50 & 7.44 & 5.26 & 6.84 & 7.61 & 6.64 & 10.27 & 7.32 \\
\hline 2000 & 4.14 & 7.97 & 8.96 & 9.58 & 11.23 & 8.38 & 4.14 & 8 & 8.74 & 9.69 & 10.97 & 8.31 \\
\hline 4000 & 3.97 & 7.57 & 7.91 & 7.05 & 9.29 & 7.16 & 3.81 & 7.39 & 7.80 & 6.91 & 9.31 & 7.04 \\
\hline Means & 4.46 & 7.51 & 8.21 & 7.77 & 10.34 & & 4.40 & 7.41 & 8.05 & 7.75 & 10.18 & \\
\hline LSD at $5 \%$ & \multicolumn{6}{|c|}{$S=0.33^{\star \star} F=0.61^{\star \star \star}(S) *(F)=1.05^{\star \star \star}$} & \multicolumn{6}{|c|}{$S=0.32^{\star \star \star} F=0.66^{\star \star \star}(S) *(F)=1.14^{\star \star}$} \\
\hline
\end{tabular}

Spraying plants with all concentrations of ascorbic acid and proline decreased significantly sodium and chloride of leaves in both seasons and cuttings. Proline at $80 \mathrm{ppm}$ gave the best results. When plants irrigated with seawater at $6000 \mathrm{ppm}$ and sprayed with 80 ppm proline, sodium of leaves decreased by 25.0 and $12.7 \%$ compared with unsprayed plants in the first and second seasons of the first cut. In addition, chloride of leaves decreased by $10.5,15 \%$.

\section{Number, length and width of stomata:}

Table (5) showed that number, length and width of stomata were decreased significantly with increasing salinity level in both cuttings in most cases in the absence of anti-stress compounds. The level of $6000 \mathrm{ppm}$ seawater gave the lowset values in the first cut and plants died before second cut when the foliar spray with ascorbic acid or proline was stoped. Spraying plants with proline and ascorbic acids increased significantly all stomata parameter. The concentration of $80 \mathrm{ppm}$ proline gave the best results $12.88,29.12$ and 24.11 for number, length and width of stomata in the first cut compared to control(9.25, 25.47 and 20.83 respectively) followed by 200 ppm ascorbic acid. 


\section{Antioxidant enzyme}

No significant differencs were found for salinity stress on the antioxidant enzymes in the both seasons and cuttings except for superoxide dismutase activity and aniline 4-hydroxylase activity in the absence of proline and ascorbic acid in the first cut of the first season as shown in Tables (6, 7, 8, 9 and 10). All concentrations of proline and ascorbic acid increased significantly most enzymatic and non enzymatic antioxidants; increased reduced glutathione (GSH), catalase activity, superoxide dismutase activity (SOD), cytochrome b5, cytochrome $\mathrm{p}_{450}$, amidopyrine N-demethylase and aniline 4-hydroxylase activity

Proline at 80 ppm followed by ascorbic acid at 200 ppm, were the most effective treatments to increase the antioxidant parameter. On the other hand, thiobarbituric acid reactive substances were decreased by proline and ascorbic acid addition. Catalse activity increased slightly with increasing salinity levels.

Table 3

Effects of ascorbic acid and proline and their interaction treatments on leaf green color degree (SPAD units) and potassium of leaves (\%) of Duranta erecta L Var. Variegata plants under salinity stress condition at 2019/2020

\begin{tabular}{|c|c|c|c|c|c|c|c|c|c|c|c|c|}
\hline \multicolumn{7}{|l|}{$2019 / 2020$ season } & \multicolumn{6}{|c|}{$2020 / 2021$ season } \\
\hline \multirow[t]{4}{*}{ Saline water level (ppm) } & \multicolumn{6}{|c|}{ Proline (P) and Ascorbic (As) concentration (ppm) } & \multicolumn{6}{|c|}{ Proline (P) and Ascorbic (As) concentration (ppm) } \\
\hline & 0.0 & As 100 & As200 & P40 & P80 & Means (S) & 0.0 & As 100 & As200 & P40 & P80 & Means (S) \\
\hline & \multicolumn{12}{|c|}{ Leaf green color degree (SPAD units) } \\
\hline & \multicolumn{6}{|c|}{ First cut } & \multicolumn{6}{|c|}{ First cut } \\
\hline 0.0 & 31.33 & 32.77 & 35.63 & 32.94 & 36.87 & 33.91 & 30.76 & 31.38 & 35.57 & 32.05 & 36.84 & 33.32 \\
\hline 2000 & 29.55 & 32.18 & 36.40 & 31.14 & 36.96 & 33.25 & 29.97 & 31.74 & 36.17 & 29.68 & 35.37 & 32.58 \\
\hline 4000 & 27.61 & 32.20 & 35.91 & 30.58 & 36.69 & 32.60 & 26.77 & 32.49 & 36.05 & 29.49 & 36.73 & 32.31 \\
\hline 6000 & 21.82 & 23.54 & 26.87 & 22.19 & 25.64 & 24.01 & 19.89 & 22.08 & 25.72 & 20.28 & 27.36 & 23.07 \\
\hline Means & 27.58 & 30.17 & 33.70 & 29.21 & 34.04 & & 26.85 & 29.42 & 33.38 & 27.87 & 34.08 & \\
\hline \multirow[t]{2}{*}{ LSD at $5 \%$} & \multicolumn{6}{|c|}{$S=2.73^{\star \star \star} F=1.86^{\star \star \star}(S) *(F)=3.71^{n s}$} & \multicolumn{6}{|c|}{$S=2.22^{\star \star \star} F=1.73^{\star \star \star}(S) *(F)=3.46^{n s}$} \\
\hline & \multicolumn{6}{|c|}{ Second cut } & \multicolumn{6}{|c|}{ Second cut } \\
\hline 0.0 & 27.84 & 30.33 & 34.29 & 30.52 & 34.66 & 31.53 & 28.71 & 28.66 & 31.08 & 28.33 & 30.98 & 29.55 \\
\hline 2000 & 27.08 & 29.55 & 33.57 & 28.84 & 34.94 & 30.80 & 27.55 & 27.72 & 30.36 & 26.55 & 28.82 & 28.20 \\
\hline 4000 & 25.47 & 26.96 & 34.00 & 28.29 & 34.56 & 29.85 & 24.07 & 25.88 & 32.22 & 27.46 & 30.99 & 28.12 \\
\hline Means & 26.80 & 28.95 & 33.95 & 29.22 & 34.72 & & 26.78 & 27.42 & 31.22 & 27.45 & 30.26 & \\
\hline \multirow[t]{3}{*}{ LSD at $5 \%$} & \multicolumn{6}{|c|}{$S=1.53^{n s} F=1.86^{\star \star *}(S) *(F)=3.23^{n s}$} & \multicolumn{6}{|c|}{$S=1.30^{n s} F=1.34^{\star * \star}(S) *(F)=2.33^{*}$} \\
\hline & \multicolumn{12}{|c|}{ Potassium of leaves (\%) } \\
\hline & \multicolumn{6}{|c|}{ First cut } & \multicolumn{6}{|c|}{ First cut } \\
\hline 0.0 & 1.53 & 1.49 & 2.55 & 1.49 & 2.70 & 1.95 & 1.69 & 1.81 & 2.62 & 1.57 & 2.71 & 2.08 \\
\hline 2000 & 1.16 & 1.47 & 2.53 & 1.57 & 2.59 & 1.86 & 1.23 & 1.62 & 2.60 & 1.14 & 2.61 & 1.84 \\
\hline 4000 & 1.02 & 1.44 & 2.46 & 1.79 & 2.53 & 1.85 & 1.08 & 1.43 & 2.27 & 1.75 & 2.53 & 1.81 \\
\hline 6000 & 1.00 & 1.26 & 1.56 & 1.31 & 1.52 & 1.33 & 1.03 & 1.29 & 1.55 & 1.60 & 1.52 & 1.40 \\
\hline Means & 1.18 & 1.42 & 2.28 & 1.54 & 2.34 & & 1.26 & 1.54 & 2.26 & 1.51 & 2.34 & \\
\hline \multirow[t]{2}{*}{ LSD at $5 \%$} & \multicolumn{6}{|c|}{$S=0.17^{\star \star \star} F=0.22^{\star \star \star}(S) *(F)=0.45^{\star}$} & \multicolumn{6}{|c|}{$S=0.24^{* \star} F=0.30^{* \star *}(S) *(F)=0.61^{n s}$} \\
\hline & \multicolumn{6}{|c|}{ Second cut } & \multicolumn{6}{|c|}{ Second cut } \\
\hline 0.0 & 2.32 & 2.62 & 2.71 & 2.18 & 2.54 & 2.47 & 2.06 & 2.13 & 2.54 & 2.09 & 2.73 & 2.31 \\
\hline 2000 & 1.27 & 1.89 & 2.60 & 1.40 & 2.43 & 1.92 & 1.17 & 3.61 & 2.34 & 2.26 & 2.60 & 2.40 \\
\hline 4000 & 1.13 & 1.48 & 2.74 & 2.11 & 2.40 & 1.97 & 1.08 & 1.45 & 2.55 & 1.71 & 2.51 & 1.86 \\
\hline Means & 1.57 & 1.99 & 2.69 & 1.90 & 2.46 & & 1.44 & 2.40 & 2.48 & 2.02 & 2.61 & \\
\hline LSD at $5 \%$ & \multicolumn{6}{|c|}{$S=0.18^{\star \star} F=0.17^{\star \star \star}(S) *(F)=0.30^{\star \star \star}$} & \multicolumn{6}{|c|}{$S=0.45^{n s} F=0.47^{\star \star \star}(S) *(F)=0.82^{\star *}$} \\
\hline
\end{tabular}


Table 4

Effects of ascorbic acid and proline and their interaction treatments on sodium of leaves (ppm) and chloride of leaves (\%) of Duranta erecta $L$ Var. Variegata plants under salinity stress condition at 2019/2020 and 2020/2021

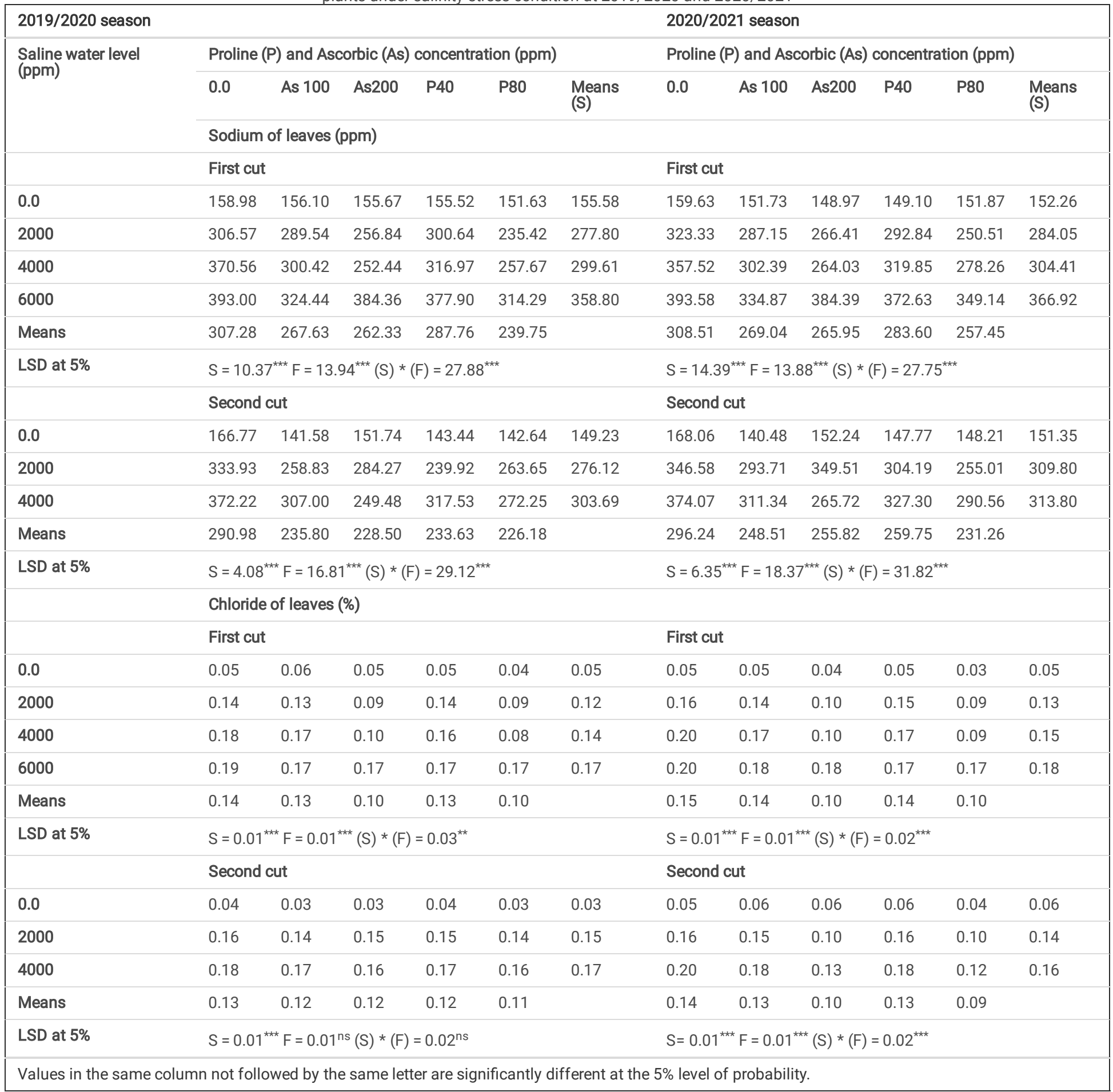


Table 5

Effects of ascorbic acid and proline and their interaction treatments on number, length and width of stomata of Duranta erecta $L$ Var. Variegata plants unc condition at 2020/2021

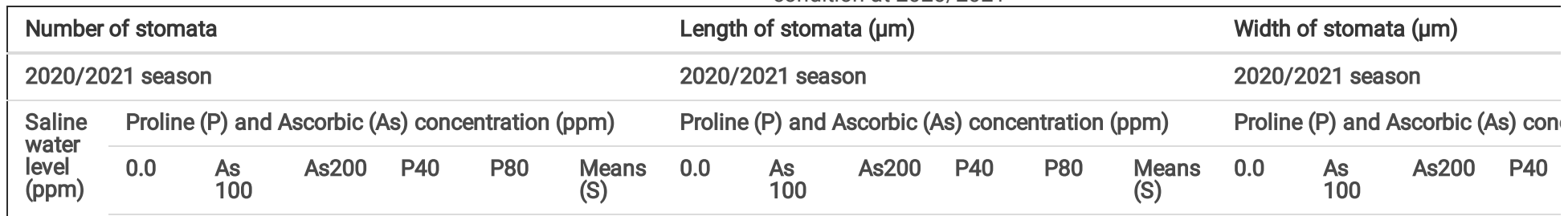

First cut

\begin{tabular}{lllllll}
0.0 & 10.50 & 11.00 & 13.50 & 11.00 & 14.00 & 12.00 \\
\hline 2000 & 10.00 & 10.50 & 13.00 & 11.00 & 12.00 & 11.30 \\
\hline 4000 & 8.50 & 10.00 & 10.50 & 10.00 & 13.50 & 10.50 \\
\hline 6000 & 8.00 & 9.00 & 11.00 & 9.00 & 12.00 & 9.80 \\
\hline Means & 9.25 & 10.13 & 12.00 & 10.25 & 12.88 &
\end{tabular}

$\underset{5 \%}{\operatorname{LSD}}$ at $S=2.28^{\mathrm{ns}} \mathrm{F}=0.93^{\star \star \star}(S) *(F)=1.87^{\mathrm{ns}}$

Second cut

$\begin{array}{llllll}\mathbf{0} .0 & 11.00 & 10.50 & 12.50 & 11.00 & 11.50\end{array}$

$\begin{array}{lllllll}2000 & 7.50 & 7.50 & 11.50 & 10.00 & 12.00 & 9.70\end{array}$

$\begin{array}{lllllll}4000 & 8.00 & 8.50 & 10.00 & 10.50 & 13.00 & 10.00\end{array}$

$\begin{array}{llllll}\text { Means } & 8.83 & 8.83 & 11.33 & 10.50 & 12.17\end{array}$

$\underset{5 \%}{\operatorname{LSD}}$ at $\quad S=1.05^{*} \mathrm{~F}=2.12^{*}(\mathrm{~S}) *(F)=3.68^{\mathrm{ns}}$
First cut

$\begin{array}{llll}29.38 & 24.38 & 30.00 & 29.80\end{array}$

$\begin{array}{llll}26.46 & 24.79 & 27.71 & 26.67\end{array}$

$23.33 \quad 24.59 \quad 29.79 \quad 26.04$

22.71

$\begin{array}{lllll}25.47 & 24.27 & 28.13 & 26.83 & 29.12\end{array}$

$S=1.80^{\star} F=2.03^{\star \star \star}(S) *(F)=4.06^{n s}$

\section{Second cut}

$\begin{array}{llllll}22.92 & 25.63 & 25.42 & 23.96 & 24.59 & 24.50\end{array}$

$\begin{array}{llllll}24.59 & 24.38 & 25.21 & 26.46 & 25.42 & 25.21\end{array}$

$\begin{array}{llllll}23.96 & 25.21 & 25.00 & 25.63 & 27.92 & 25.54\end{array}$

$\begin{array}{lllll}23.82 & 25.07 & 25.21 & 25.35 & 25.97\end{array}$

$S=3.43^{n s} F=1.75^{n s}(S) *(F)=3.04^{n s}$
First cut

$23.54 \quad 23.13 \quad 25.83 \quad 24.59$

$\begin{array}{llll}21.46 & 21.04 & 24.79 & 20.63\end{array}$

$\begin{array}{llll}19.58 & 20.00 & 26.05 & 20.63\end{array}$

$\begin{array}{llll}18.75 & 19.59 & 21.05 & 20.21\end{array}$

$\begin{array}{llll}20.83 & 20.94 & 24.43 & 21.51\end{array}$

$S=1.60^{\star} F=1.45^{\star \star \star}(S) *(F)=2.91$

\section{Second cut}

$\begin{array}{llll}19.80 & 21.67 & 21.04 & 20.63\end{array}$

$\begin{array}{llll}19.79 & 21.04 \quad 21.88 \quad 21.46\end{array}$

$21.25 \quad 22.71 \quad 22.71 \quad 21.8 \varepsilon$

$20.28 \quad 21.81 \quad 21.88 \quad 21.32$

$S=2.80^{\text {ns }} F=1.36^{\text {ns }}(S) *(F)=2 .$.

Values in the same column not followed by the same letter are significantly different at the $5 \%$ level of probability.

Table 6

Effects of ascorbic acid, proline and their interaction treatments on thiobarbituric acid reactive substances (TBARS) nmole/g of Duranta erecta $L$ Var. Variegata plants under salinity stress condition at 2019/2020

\begin{tabular}{|c|c|c|c|c|c|c|c|c|c|c|c|c|}
\hline \multicolumn{7}{|l|}{$2019 / 2020$ season } & \multicolumn{6}{|c|}{ 2020/2021 season } \\
\hline \multirow[t]{3}{*}{ Saline water level (ppm) } & \multicolumn{6}{|c|}{ Proline (P) and Ascorbic (As) concentration (ppm) } & \multicolumn{6}{|c|}{ Proline (P) and Ascorbic (As) concentration (ppm) } \\
\hline & 0.0 & As 100 & As200 & P40 & P80 & Means (S) & 0.0 & As 100 & As200 & P40 & P80 & Means (S) \\
\hline & \multicolumn{6}{|c|}{ First cut } & \multicolumn{6}{|c|}{ First cut } \\
\hline 0.0 & 9.26 & 8.94 & 9.08 & 8.80 & 9.22 & 9.06 & 9.30 & 9.08 & 9.03 & 8.91 & 9.27 & 9.12 \\
\hline 2000 & 9.73 & 8.65 & 9.08 & 9.04 & 7.62 & 8.82 & 9.80 & 9.85 & 9.16 & 9.08 & 7.54 & 9.08 \\
\hline 4000 & 9.93 & 8.89 & 8.84 & 8.93 & 7.36 & 8.79 & 9.97 & 8.92 & 8.90 & 9.01 & 7.35 & 8.83 \\
\hline 6000 & 9.54 & 8.70 & 8.83 & 8.72 & 9.23 & 9.00 & 9.39 & 8.60 & 8.75 & 8.66 & 9.26 & 8.93 \\
\hline Means & 9.61 & 8.79 & 8.96 & 8.87 & 8.36 & & 9.61 & 9.11 & 8.96 & 8.91 & 8.35 & \\
\hline \multirow[t]{2}{*}{ LSD at $5 \%$} & \multicolumn{6}{|c|}{$S=1.17^{n s} F=1.65^{n s}(S) *(F)=2.68^{n s}$} & \multicolumn{6}{|c|}{$S=1.46^{\mathrm{ns}} F=2.07^{\mathrm{ns}}(S) *(F)=2.19^{\mathrm{ns}}$} \\
\hline & \multicolumn{6}{|c|}{ Second cut } & \multicolumn{6}{|c|}{ Second cut } \\
\hline 0.0 & 9.27 & 9.14 & 9.02 & 8.97 & 9.32 & 9.14 & 9.20 & 9.00 & 8.98 & 8.82 & 9.34 & 9.07 \\
\hline 2000 & 9.82 & 8.66 & 9.19 & 9.06 & 6.32 & 8.61 & 9.85 & 8.61 & 8.87 & 8.97 & 7.59 & 8.78 \\
\hline 4000 & 10.02 & 8.93 & 9.05 & 8.87 & 7.50 & 8.61 & 10.08 & 8.92 & 8.85 & 8.94 & 7.35 & 8.83 \\
\hline Means & 9.70 & 8.91 & 9.09 & 8.97 & 7.71 & & 9.71 & 8.84 & 8.90 & 8.91 & 8.09 & \\
\hline LSD at $5 \%$ & \multicolumn{6}{|c|}{$S=1.89^{n s} F=2.31^{*}(S) *(F)=2.06^{n s}$} & \multicolumn{6}{|c|}{$S S=1.57^{n s} F=1.92^{n s}(S) *(F)=2.23^{n s}$} \\
\hline
\end{tabular}


Table 7

Effects of ascorbic acid and proline and their interaction treatments on reduced glutathione (GSH) $\mathrm{mg} / \mathrm{g}$ tissue and catalase activity (IU/g tissue) of Duranta erecta L Var. Variegata plants under salinity stress condition at 2019/2020 and 2020/2021

\begin{tabular}{|c|c|c|c|c|c|c|c|c|c|c|c|c|}
\hline \multicolumn{7}{|l|}{$2019 / 2020$ season } & \multicolumn{6}{|c|}{$2020 / 2021$ season } \\
\hline \multirow[t]{4}{*}{ Saline water level (ppm) } & \multicolumn{6}{|c|}{ Proline (P) and Ascorbic (As) concentration (ppm) } & \multicolumn{6}{|c|}{ Proline (P) and Ascorbic (As) concentration (ppm) } \\
\hline & 0.0 & As 100 & As200 & P40 & P80 & Means (S) & 0.0 & As 100 & As200 & P40 & P80 & Means (S) \\
\hline & \multicolumn{12}{|c|}{ Reduced glutathione (GSH) $\mathrm{mg} / \mathrm{g}$} \\
\hline & \multicolumn{6}{|c|}{ First cut } & \multicolumn{6}{|c|}{ First cut } \\
\hline 0.0 & 21.11 & 28.80 & 39.33 & 34.18 & 43.46 & 33.37 & 20.53 & 28.31 & 38.66 & 33.60 & 42.80 & 32.78 \\
\hline 2000 & 20.40 & 30.80 & 40.93 & 33.78 & 42.66 & 33.71 & 19.86 & 30.71 & 40.80 & 34.31 & 43.20 & 33.77 \\
\hline 4000 & 21.51 & 30.53 & 39.78 & 34.49 & 42.71 & 33.80 & 21.20 & 30.00 & 38.81 & 34.80 & 42.58 & 33.47 \\
\hline 6000 & 20.00 & 26.40 & 39.20 & 27.20 & 39.64 & 30.48 & 19.69 & 26.49 & 38.58 & 27.51 & 38.93 & 30.24 \\
\hline Means & 20.75 & 29.13 & 39.81 & 32.41 & 42.12 & & 20.32 & 28.88 & 39.21 & 32.55 & 41.87 & \\
\hline \multirow[t]{2}{*}{ LSD at $5 \%$} & \multicolumn{6}{|c|}{$S=6.30^{n s} F=4.01^{\star \star \star}(S) *(F)=8.02^{n s}$} & \multicolumn{6}{|c|}{$S=6.32^{\mathrm{ns}} F=3.96^{\star \star \star}(S) *(F)=7.92^{\mathrm{ns}}$} \\
\hline & \multicolumn{6}{|c|}{ Second cut } & \multicolumn{6}{|c|}{ Second cut } \\
\hline 0.0 & 21.38 & 29.20 & 39.60 & 34.40 & 43.73 & 33.66 & 21.38 & 29.38 & 39.99 & 35.24 & 43.78 & 33.95 \\
\hline 2000 & 20.71 & 30.80 & 41.20 & 34.00 & 43.11 & 34.00 & 20.66 & 31.06 & 41.24 & 34.00 & 43.64 & 34.12 \\
\hline 4000 & 21.78 & 31.06 & 40.31 & 34.80 & 42.26 & 34.04 & 21.60 & 30.98 & 39.46 & 34.40 & 42.13 & 33.71 \\
\hline Means & 21.29 & 30.41 & 40.37 a & 34.40 & 43.03 & & 21.21 & 30.47 & 40.23 & 34.54 & 43.18 & \\
\hline \multirow[t]{3}{*}{ LSD at $5 \%$} & \multicolumn{6}{|c|}{$S=10.52^{n s} F=5.40^{\star \star \star}(S) *(F)=9.35^{n s}$} & \multicolumn{6}{|c|}{$S=9.66^{n s} F=5.11^{\star \star \star}(S) *(F)=8.86^{n s}$} \\
\hline & \multicolumn{12}{|c|}{ Catalase activity (IU/g tissue) } \\
\hline & \multicolumn{6}{|c|}{ First cut } & \multicolumn{6}{|c|}{ First cut } \\
\hline 0.0 & 8.20 & 11.84 & 17.60 & 17.99 & 19.14 & 14.95 & 8.51 & 12.91 & 18.72 & 18.96 & 20.70 & 15.96 \\
\hline 2000 & 8.60 & 11.29 & 16.31 & 16.00 & 17.08 & 13.85 & 9.39 & 11.80 & 17.27 & 17.38 & 18.38 & 14.84 \\
\hline 4000 & 9.40 & 10.34 & 16.99 & 16.28 & 19.74 & 14.55 & 11.13 & 11.27 & 17.95 & 17.24 & 20.35 & 15.59 \\
\hline 6000 & 11.57 & 12.80 & 13.07 & 11.84 & 15.58 & 12.97 & 12.37 & 13.87 & 14.15 & 13.45 & 16.81 & 14.13 \\
\hline Means & 9.44 & 11.57 & 15.99 & 15.52 & 17.88 & & 10.35 & 12.46 & 17.02 & 16.75 & 19.06 & \\
\hline \multirow[t]{2}{*}{ LSD at $5 \%$} & \multicolumn{6}{|c|}{$S=1.70^{n s} F=1.90^{\star \star \star}(S) *(F)=3.80^{n s}$} & \multicolumn{6}{|c|}{$S=1.56^{n s} F=1.96^{\star \star \star}(S) *(F)=3.92^{n s}$} \\
\hline & \multicolumn{6}{|c|}{ Second cut } & \multicolumn{6}{|c|}{ Second cut } \\
\hline 0.0 & 8.04 & 11.92 & 16.96 & 17.63 & 18.49 & 14.60 & 8.48 & 9.85 & 16.32 & 18.85 & 19.74 & 14.65 \\
\hline 2000 & 9.01 & 11.38 & 16.80 & 15.65 & 17.06 & 13.98 & 8.12 & 9.85 & 17.19 & 12.53 & 17.69 & 13.07 \\
\hline 4000 & 9.28 & 11.02 & 17.20 & 17.39 & 19.95 & 14.97 & 8.85 & 12.46 & 15.84 & 17.19 & 19.55 & 14.78 \\
\hline Means & 8.77 & 11.44 & 16.98 & 16.89 & 18.50 & & 8.48 & 10.72 & 16.45 & 16.19 & 18.99 & \\
\hline LSD at $5 \%$ & \multicolumn{6}{|c|}{$S=3.29^{n s} F=2.24^{\star \star *}(S) *(F)=3.88^{n s}$} & \multicolumn{6}{|c|}{$S=3.99^{n s} F=1.54^{\star \star \star}(S) *(F)=2.66^{*}$} \\
\hline
\end{tabular}


Table 8

Effects of ascorbic acid, proline and their interaction treatments on superoxide dismutase activity (SOD) IU/g tissue of Duranta erecta $L$ Var. Variegata plants under salinity stress condition at 2019/2020 and 2020/2021

\begin{tabular}{|c|c|c|c|c|c|c|c|c|c|c|c|c|}
\hline \multicolumn{7}{|c|}{$2019 / 2020$ season } & \multicolumn{6}{|c|}{$2020 / 2021$ season } \\
\hline \multirow{3}{*}{$\begin{array}{l}\text { Saline water } \\
\text { level (ppm) }\end{array}$} & \multicolumn{6}{|c|}{ Proline (P) and Ascorbic (As) concentration (ppm) } & \multicolumn{6}{|c|}{ Proline (P) and Ascorbic (As) concentration (ppm) } \\
\hline & 0.0 & As 100 & As200 & P40 & P80 & $\begin{array}{l}\text { Means } \\
\text { (S) }\end{array}$ & 0.0 & As 100 & As200 & P40 & P80 & $\begin{array}{l}\text { Means } \\
(S)\end{array}$ \\
\hline & \multicolumn{6}{|l|}{ First cut } & \multicolumn{6}{|l|}{ First cut } \\
\hline 0.0 & 1412.11 & 1423.83 & 1453.13 & 1435.55 & 1458.99 & 1436.72 & 1400.39 & 1417.97 & 1435.55 & 1429.69 & 1453.13 & 1427.30 \\
\hline 2000 & 1417.97 & 1429.69 & 1453.13 & 1447.27 & 1453.13 & 1440.24 & 1406.25 & 1423.83 & 1406.26 & 1429.69 & 1447.27 & 1422.60 \\
\hline 4000 & 1400.39 & 1429.69 & 1458.99 & 1435.55 & 1464.85 & 1437.89 & 1406.25 & 1376.96 & 1458.99 & 1441.41 & 1476.41 & 1432.00 \\
\hline 6000 & 1388.67 & 1412.11 & 1423.82 & 1417.97 & 1435.54 & 1415.62 & 1347.66 & 1371.10 & 1412.11 & 1435.55 & 1423.82 & 1398.00 \\
\hline Means & 1404.79 & 1423.83 & 1447.27 & 1434.09 & 1453.12 & & 1390.14 & 1397.46 & 1428.23 & 1434.09 & 1450.15 & \\
\hline \multirow[t]{2}{*}{ LSD at $5 \%$} & \multicolumn{6}{|c|}{$S=6.81^{* \star} F=12.18^{* \star *}(S) *(F)=24.37^{n s}$} & \multicolumn{6}{|c|}{$S=72.37^{n s} F=26.49^{* \star *}(S) *(F)=52.98^{n s}$} \\
\hline & \multicolumn{6}{|c|}{ Second cut } & \multicolumn{6}{|c|}{ Second cut } \\
\hline 0.0 & 1394.53 & 1417.97 & 1441.41 & 1423.83 & 1441.41 & 1423.83 & 1406.25 & 1423.83 & 1458.99 & 1429.69 & 1447.27 & 1433.20 \\
\hline 2000 & 1394.53 & 1412.11 & 1400.40 & 1406.25 & 1441.41 & 1410.94 & 1406.25 & 1417.97 & 1429.69 & 1423.83 & 1447.27 & 1425.00 \\
\hline 4000 & 1353.52 & 1388.68 & 1447.27 & 1453.13 & 1476.56 & 1423.83 & 1347.66 & 1388.68 & 1447.27 & 1447.27 & 1464.85 & 1419.10 \\
\hline Means & 1380.86 & 1406.25 & 1429.69 & 1427.74 & 1453.13 & & 1386.72 & 1410.16 & 1445.31 & 1433.60 & 1453.13 & \\
\hline LSD at $5 \%$ & \multicolumn{6}{|c|}{$S=17.94^{\mathrm{ns}} F=45.91^{*}(S) *(F)=79.52^{\mathrm{ns}}$} & \multicolumn{6}{|c|}{$S=46.39^{n s} F=42.34^{*}(S) *(F)=73.34^{n s}$} \\
\hline
\end{tabular}


Table 9

Effects of ascorbic acid and proline and their interaction treatments on cytochrome b5 and cytochrome P450 (CYP450) nmol cytochrome/mg protein of Duranta erecta L Var. Variegata plants under salinity stress condition at 2019/2020 and 2020/2021

\begin{tabular}{|c|c|c|c|c|c|c|c|c|c|c|c|c|}
\hline \multicolumn{7}{|l|}{$2019 / 2020$ season } & \multicolumn{6}{|c|}{$2020 / 2021$ season } \\
\hline \multirow[t]{4}{*}{ Saline water level (ppm) } & \multicolumn{6}{|c|}{ Proline (P) and Ascorbic (As) concentration (ppm) } & \multicolumn{6}{|c|}{ Proline (P) and Ascorbic (As) concentration (ppm) } \\
\hline & 0.0 & As 100 & As200 & P40 & P80 & Means (S) & 0.0 & As 100 & As200 & P40 & P80 & Means (S) \\
\hline & \multicolumn{12}{|c|}{ Cytochrome b5 nmol cytochrome/mg protein } \\
\hline & \multicolumn{6}{|c|}{ First cut } & \multicolumn{6}{|c|}{ First cut } \\
\hline 0.0 & 0.70 & 1.04 & 1.34 & 1.39 & 1.42 & 1.18 & 0.60 & 1.02 & 1.70 & 1.35 & 1.40 & 1.21 \\
\hline 2000 & 0.60 & 1.08 & 1.37 & 1.40 & 1.41 & 1.17 & 0.58 & 1.04 & 1.60 & 1.38 & 1.55 & 1.20 \\
\hline 4000 & 0.63 & 1.17 & 1.57 & 1.40 & 1.43 & 1.24 & 0.61 & 0.97 & 1.55 & 1.22 & 1.60 & 1.16 \\
\hline 6000 & 0.56 & 1.22 & 1.42 & 1.38 & 1.45 & 1.20 & 0.64 & 1.12 & 1.48 & 1.37 & 1.38 & 1.20 \\
\hline Means & 0.62 & 1.12 & 1.42 & 1.39 & 1.43 & & 0.61 & 1.04 & 1.58 & 1.33 & 1.40 & \\
\hline \multirow[t]{2}{*}{ LSD at $5 \%$} & \multicolumn{6}{|c|}{$S=0.47^{n s} F=0.24^{\star \star \star}(S) *(F)=0.49^{n s}$} & \multicolumn{6}{|c|}{$S=0.18^{n s} F=0.26^{\star \star *}(S) *(F)=0.53^{n s}$} \\
\hline & \multicolumn{6}{|c|}{ Second cut } & \multicolumn{6}{|c|}{ Second cut } \\
\hline 0.0 & 0.70 & 1.06 & 1.38 & 1.38 & 1.42 & 1.18 & 0.61 & 1.06 & 1.41 & 0.95 & 1.89 & 1.18 \\
\hline 2000 & 0.54 & 1.09 & 1.45 & 1.42 & 1.39 & 1.18 & 0.57 & 1.02 & 1.47 & 1.24 & 1.90 & 1.24 \\
\hline 4000 & 0.63 & 1.13 & 1.49 & 1.10 & 1.40 & 1.15 & 0.59 & 1.17 & 1.56 & 1.32 & 1.90 & 1.31 \\
\hline Means & 0.62 & 1.09 & 1.44 & 1.30 & 1.40 & & 0.59 & 1.08 & 1.48 & 1.17 & 1.90 & \\
\hline \multirow[t]{3}{*}{ LSD at $5 \%$} & \multicolumn{6}{|c|}{$S=0.92^{\mathrm{ns}} F=0.23^{\star \star \star}(S) *(F)=0.41^{\mathrm{ns}}$} & \multicolumn{6}{|c|}{$S=0.37^{n s} F=0.17^{* \star *}(S) *(F)=0.30^{n s}$} \\
\hline & \multicolumn{12}{|c|}{ Cytochrome P450 (CYP450) nmol cytochrome/mg protein } \\
\hline & \multicolumn{6}{|c|}{ First cut } & \multicolumn{6}{|c|}{ First cut } \\
\hline 0.0 & 1.62 & 1.83 & 2.27 & 1.94 & 2.66 & 2.06 & 1.51 & 1.71 & 2.35 & 2.03 & 2.38 & 1.99 \\
\hline 2000 & 1.53 & 2.03 & 2.33 & 1.95 & 2.61 & 2.09 & 1.44 & 1.75 & 2.30 & 2.11 & 2.52 & 2.02 \\
\hline 4000 & 1.42 & 1.60 & 2.22 & 1.64 & 2.53 & 1.88 & 1.33 & 1.64 & 2.34 & 1.78 & 2.74 & 1.96 \\
\hline 6000 & 1.43 & 1.42 & 2.30 & 2.08 & 2.39 & 1.92 & 1.50 & 1.72 & 2.43 & 2.18 & 2.47 & 2.06 \\
\hline Means & 1.50 & 1.72 & 2.28 & 1.90 & 2.55 & & 1.44 & 1.70 & 2.35 & 2.02 & 2.53 & \\
\hline \multirow[t]{2}{*}{ LSD at $5 \%$} & \multicolumn{6}{|c|}{$S=0.33^{n s} F=0.37^{* \star *}(S) *(F)=0.74^{n s}$} & \multicolumn{6}{|c|}{$S=0.39^{n s} F=0.36^{* * *}(S) *(F)=0.72^{n s}$} \\
\hline & \multicolumn{6}{|c|}{ Second cut } & \multicolumn{6}{|c|}{ Second cut } \\
\hline 0.0 & 1.39 & 1.75 & 2.23 & 1.37 & 2.52 & 1.85 & 1.16 & 1.53 & 1.93 & 1.91 & 2.44 & 1.80 \\
\hline 2000 & 1.54 & 1.94 & 2.23 & 2.12 & 2.55 & 2.07 & 1.38 & 1.32 & 2.16 & 2.11 & 2.22 & 1.84 \\
\hline 4000 & 1.12 & 1.34 & 2.17 & 1.23 & 2.74 & 1.72 & 1.38 & 1.92 & 2.26 & 1.91 & 2.54 & 2.00 \\
\hline Means & 1.35 & 1.67 & 2.21 & 1.57 & 2.60 & & 1.31 & 1.59 & 2.11 & 1.98 & 2.40 & \\
\hline LSD at $5 \%$ & \multicolumn{6}{|c|}{$S=1.12^{n s} F=0.41^{\star \star \star}(S) *(F)=0.70^{n s}$} & \multicolumn{6}{|c|}{$S=0.33^{n s} F=0.35^{\star \star \star}(S) *(F)=0.60^{n s}$} \\
\hline
\end{tabular}


Table 10

Effects of ascorbic acid and proline and their interaction treatments on amidopyrine $\mathrm{N}$-demethylase $\mu \mathrm{mol} / \mathrm{min} \times \mathrm{kg}$ (plant tissue) and aniline 4hydroxylase activity $\mu \mathrm{mol} / \mathrm{min} / \mathrm{mg}$ protein of Duranta erecta $L$ Var. Variegata plants under salinity stress condition at $2019 / 2020$ and $2020 / 2021$

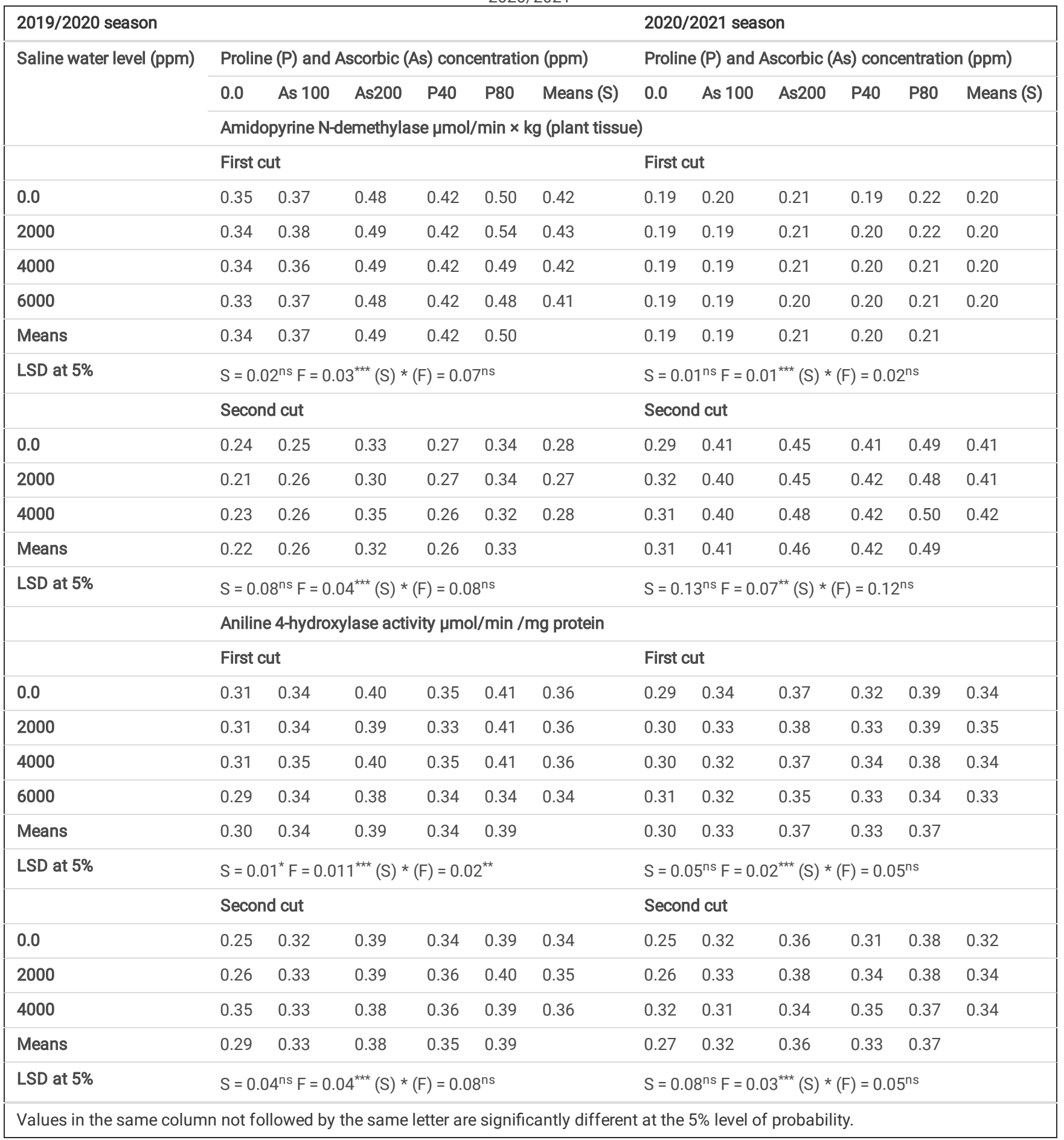

\section{DNA breakage and Plant survival \%}

As shown in Table (11) the highest level of seawater $(6000 \mathrm{ppm})$ slightly increased DNA breakages $\%$ in both cutting of the first season. On the other hand, all concentrarions of ascorbic acid and proline decreased the percentage of DNA breakages in both seasons and cuttings. Proline at 80 ppm and ascorbic acid at 200 ppm were the best.

Salinity irrigation at 4000 and 6000 ppm decreased significantly plant survival (\%) in both cuttings and seasons. The plant survival decreased by 66.7 and $75.0 \%$ in the first and second seasons respectively when plant irrigated by seawater at $6000 \mathrm{ppm}$ without spraying with proline or ascorbic acid. While, spraying plants with all concentrations of proline and ascorbic acid increased significantly plant survival (\%). Proline treatments at 80 ppm increased survival $\%$ for plant that irrigated by seawater at 6000 ppm by 75 and $133 \%$ in the first cut of the first and second seasons respectively. The increased reached to $200.0 \%$ for plants irrigated by seawater at $4000 \mathrm{ppm}$ in the second cut of the both seasons 
Table 11

Effects of ascorbic acid and proline and their interaction treatments on DNA breakages (\%) and plant survival (\%) of Duranta erecta $L$ Var. Variegata plants under salinity stress condition at 2019/2020 and 2020/2021.

\begin{tabular}{|c|c|c|c|c|c|c|c|c|c|c|c|c|}
\hline \multicolumn{7}{|l|}{$2019 / 2020$ season } & \multicolumn{6}{|c|}{$2020 / 2021$ season } \\
\hline \multirow{4}{*}{$\begin{array}{l}\text { Saline water level } \\
\text { (ppm) }\end{array}$} & \multicolumn{6}{|c|}{ Proline (P) and Ascorbic (As) concentration (ppm) } & \multicolumn{6}{|c|}{ Proline (P) and Ascorbic (As) concentration (ppm) } \\
\hline & 0.0 & As 100 & As200 & P40 & P80 & $\begin{array}{l}\text { Means } \\
\text { (S) }\end{array}$ & 0.0 & As 100 & As200 & P40 & P80 & $\begin{array}{l}\text { Means } \\
\text { (S) }\end{array}$ \\
\hline & \multicolumn{12}{|c|}{ DNA breakages (\%) } \\
\hline & \multicolumn{6}{|c|}{ First cut } & \multicolumn{6}{|l|}{ First cut } \\
\hline 0.0 & 9.40 & 5.37 & 5.68 & 5.33 & 7.77 & 6.71 & 9.31 & 7.24 & 5.84 & 5.57 & 7.80 & 7.15 \\
\hline 2000 & 8.30 & 8.07 & 4.28 & 4.57 & 6.86 & 6.41 & 8.93 & 8.48 & 4.30 & 4.72 & 6.68 & 6.62 \\
\hline 4000 & 8.41 & 6.02 & 5.78 & 7.04 & 3.23 & 6.09 & 7.82 & 5.08 & 5.90 & 6.97 & 3.34 & 5.82 \\
\hline 6000 & 8.86 & 8.45 & 8.29 & 7.31 & 6.47 & 7.87 & 8.27 & 8.37 & 8.29 & 7.36 & 6.50 & 7.76 \\
\hline Means & 8.74 & 6.98 & 6.01 & 6.06 & 6.08 & & 8.58 & 7.29 & 6.08 & 6.15 & 6.08 & \\
\hline \multirow[t]{2}{*}{ LSD at $5 \%$} & \multicolumn{6}{|c|}{$S=2.90^{n s} F=1.81^{*}(S) *(F)=3.62^{n s}$} & \multicolumn{6}{|c|}{$S=2.53^{n s} F=1.96^{n s}(S) *(F)=3.93^{n s}$} \\
\hline & \multicolumn{6}{|c|}{ Second cut } & \multicolumn{6}{|c|}{ Second cut } \\
\hline 0.0 & 9.77 & 7.02 & 4.42 & 6.35 & 8.88 & 7.29 & 9.79 & 7.11 & 4.66 & 6.37 & 8.92 & 7.37 \\
\hline 2000 & 8.05 & 7.67 & 5.601 & 9.52 & 4.19 & 7.01 & 8.20 & 7.72 & 5.78 & 9.54 & 4.48 & 7.14 \\
\hline 4000 & 7.97 & 6.06 & 5.99 & 6.82 & 6.23 & 6.61 & 7.99 & 6.45 & 6.06 & 6.82 & 6.27 & 6.72 \\
\hline Means & 8.59 & 6.92 & 5.34 & 7.56 & 6.43 & & 8.66 & 7.09 & 5.50 & 7.57 & 6.56 & \\
\hline \multirow[t]{3}{*}{ LSD at $5 \%$} & \multicolumn{6}{|c|}{$S=4.10^{n s} F=2.69^{n s}(S) *(F)=4.66^{n s}$} & \multicolumn{6}{|c|}{$S=4.13^{n s} F=2.70^{n s}(S) *(F)=4.68^{n s}$} \\
\hline & \multicolumn{12}{|c|}{ Plant survival (\%) } \\
\hline & \multicolumn{6}{|c|}{ First cut } & \multicolumn{6}{|l|}{ First cut } \\
\hline 0.0 & 100.00 & 100.00 & 100.00 & 100.00 & 100.00 & 100.00 & 100.00 & 100.00 & 100.00 & 100.00 & 100.00 & 100.00 \\
\hline 2000 & 100.00 & 100.00 & 100.00 & 100.00 & 100.00 & 100.00 & 100.00 & 100.00 & 100.00 & 100.00 & 100.00 & 100.00 \\
\hline 4000 & 66.67 & 83.33 & 100.00 & 91.67 & 100.00 & 88.33 & 58.33 & 83.33 & 100.00 & 91.67 & 100.00 & 86.67 \\
\hline 6000 & 33.33 & 33.33 & 50.00 & 50.00 & 58.33 & 45.00 & 25.00 & 33.33 & 50.00 & 33.33 & 58.33 & 40.00 \\
\hline Means & 75.00 & 79.17 & 87.50 & 85.42 & 89.58 & & 70.83 & 79.17 & 87.50 & 81.25 & 89.58 & \\
\hline \multirow[t]{2}{*}{ LSD at $5 \%$} & \multicolumn{6}{|c|}{$S=13.21^{\star \star \star} F=5.53^{\star \star \star}(S) *(F)=11.07^{\star \star}$} & \multicolumn{6}{|c|}{$S=9.56^{\star \star \star} F=5.53^{\star \star \star}(S) *(F)=11.07^{\star \star \star}$} \\
\hline & \multicolumn{6}{|c|}{ Second cut } & \multicolumn{6}{|c|}{ Second cut } \\
\hline 0.0 & 100.00 & 100.00 & 100.00 & 100.00 & 100.00 & 100.00 & 100.00 & 100.00 & 100.00 & 100.00 & 100.00 & 100.00 \\
\hline 2000 & 100.00 & 100.00 & 100.00 & 100.00 & 100.00 & 100.00 & 100.00 & 100.00 & 100.00 & 100.00 & 100.00 & 100.00 \\
\hline 4000 & 33.33 & 66.67 & 100.00 & 83.33 & 100.00 & 76.67 & 33.33 & 83.33 & 100.00 & 66.67 & 100.00 & 76.67 \\
\hline Means & 77.78 & 88.88 & 100.00 & 94.44 & 100.00 & & 77.78 & 94.44 & 100.00 & 88.89 & 100.00 & \\
\hline LSD at $5 \%$ & \multicolumn{6}{|c|}{$S=19.99^{\star} F=10.26^{\star \star \star}(S) *(F)=17.76^{\star \star \star}$} & \multicolumn{6}{|c|}{$S=7.56^{\star \star} F=13.57^{\star}(S) *(F)=23.50^{\star \star}$} \\
\hline
\end{tabular}

\section{Discussion}

The application of proline to the leaves is a shotgun strategy to reducing the negative impacts of salt. Ascorbic acid and proline enhanced plant growth, they increasd plant height and leaves dry weight of Duranta under salinity stress. In addition, Siddiqueet al. (2015) found that foliar proline administration resulted in a considerable boost in rice plant growth metrics. Buttet al. (2016) discovered that application of proline under salinity stress conditions increased plant height of chilli plants. Radyet al. (2016) established that application of proline of levels (3,6 or $9 \mathrm{mM}$ ) to lupine types resulted in a significant boost in growth characteristics as compared to the control. Maiaet al. (2017) and Soltaniet al. (2014) reported that using some foliar applications such as ascorbic acid and proline gave better various photosynthetic and inhanced plant growth and productivity under saline irrigation water treatments. Moreover, the decrease in photosynthesis and growth is caused by the increase of free radicals (ROS) in chloroplasts and the breakdown of chlorophyll molecules under salinity stress (Sadaket al. 2020). This increase in chlorophyll content could be owing to larger levels of amino acids being available to the treated plants, as amino acids aid to boost chlorophyll content, which could lead to an increase in certain growth parameters (Awadet al. 2007). In similar studies Alotaibiet al. (2019) showed 
the enhanced chlorophyll content in leaves on Simmondsia chinensis (Link) Schneider, Abd-El-Hadyet al. (2019) on Acalypha Wilkesiana, Malkiewicz and Smolik. (2019) on Petuniaxatkinsiana any further reduction in stomatal conductance and photosynthetic rate inhibit plant growth and reduce productivity (Tester and Davenport 2003). Bosabalidis and Kofidis (2002) discovered plants anatomical changes, particularly in their leaves, which are the plant's major organs for water loss. Changes in cuticle thickness, stomata density, non-glandular scales, epidermal and mesophyll cells are examples of such adaptations to preserve water under stress conditions. The stomatal conductance is linked with a water use efficiency of the plants, closing the stomata during most of the day, avoiding water loss by transpiration. As a result of the current investigation, it can be concluded that exogenous proline administration increased stomatal conductance by maintaining sufficient cellular turgor (Kamranet al., 2009), allowing for greater sub-stomatal accumulation and CO2 absorption. Ramadanet al. (2019) stated that Salt stress alters the availability, competitive absorption, and translocation of nutrients to plant parts. Plant nutrition is unbalanced as a result of other components interfering with excessive concentrations of $\mathrm{Na}$ and $\mathrm{Cl}$ ions in the soil. The increase in $\mathrm{Na}+$ absorption caused by salinity was accompanied by a decrease in $\mathrm{K}+$ concentration, indicating an apparent antagonism relationship between $\mathrm{K}+$ and $\mathrm{Na}+(\mathrm{Cuinet}$ al. 2009). Spraying with Proline reduces sodium absorption and enhances potassium absorption (Abd El-Samad 2010) on maize. On the other hand, Ahmed (1996) concluded that, application of ascorbic acid enhancement the amount of organic acids excreted into the soil by the roots, hence increasing the solubility of most nutrients that dissolve slowly into the rhizosphere zone. These study agreement with Wuet al. (2017) and lbrahimet al. (2019) who discussed the application of proline to the leaves is strategy to reducing the negative impacts of salt. From the previous results, it is clear that application of exogenous proline reduces sodium absorption and the toxicity caused by salt, and this is consistent with Alotaibiet al. (2019), Husseinet al. (2017). Water stress enhanced TBARS accumulation, which was partially prevented when plants were given exogenous ascorbic acid and proline as was the case with salt stress. The level of osmotic stress was too Iwo allow to a parallel of the effects on plant survival. Several data suggested that the salt-induced transition to ermanent wilting was linked to increased cellular activity of potentially harmful active oxygen species (AOS). Ascorbic acid scavenged reactive oxygen species and inhibited protein oxidation and degradation, according to Dolatabadianet al. (2010) Non-enzymatic antioxidant mechanisms such as GSH responded differently to the adverse effect treatment of $\mathrm{NaCl}$ (Jaleelet al. 2007). Endogenous ascorbate at a high level is required to adequately sustain the antioxidant system that protects plants from oxidative damage caused by abiotic stress. GSH is a nonenzymatic antioxidant that protects proteins containing sulfhydryl groups, participates in amino acid membrane transport, and increases plant stress tolerance (Zhaoet al. 2019). Proline contributes to the maintenance of the glutathione pool in the reduced state, which is used by dehydroascorbate reductase and other GSH-dependent enzymes involved in the antioxidant defense and glyoxalase systems (Hoqueet al. 2008). Proline also has a regulatory role in the activity and function of CAT enzymes in plant cells, as well as their engagement in metabolic response development in response to environmental stimuli, according to Abdallah and El- Bassiouny (2016). The antioxidant enzyme system (the first line of defence) protects stressed plant cells from oxidative damage by scavenging active oxygen species $\left(\mathrm{O}^{-}, \mathrm{OH}^{-}\right.$, and $\left.\mathrm{H}_{2} \mathrm{O}_{2}\right)$ within the cells. Foliar application of ascorbic acid in general, significantly increased the all enzyme activities as compared to the corresponding salinity levels. Ascorbic acid serves as a main substrate in the cyclic pathway for enzyme detoxification of hydrogen peroxide (Shalata and Neumann 2001). SOD reduced ROS production, thus reducing cell oxidation, these results are agreement with Solimanet al. (2015).

In this study, treated plants with ascorbic acid and proline increased cytochrome b5 in leaves. The enzyme is a component of an ER-associated redox chain that delivers electrons to cytochrome b5, an intermediate electron donor in a variety of lipid modification processes including as NADH-dependent fatty acid desaturation, sterol precursor desaturation, and fatty acid hydroxylation (Rahier et al. 1997). Spraying plants with proline and ascorbic acid increased CYP450. CYP450 is a plant enzyme that is involved in the manufacture of secondary metabolites such as phenylpropanoids, terpenoids, alkaloids, and lignins, as well as signalling molecules, fatty acids, lipids, defense-related chemicals, and plant hormones as reported by Goujon et al. (2003). However, pretreatment with proline and ascorbic acid reduced the effect of free radicals and helped maintain levels of the enzymes such as amidopyrine $\mathrm{N}$-demethylase in plants. Amidopyrine $\mathrm{N}$-demethylase is an enzyme of the cytochrome P450 that helps in the metabolism of cells as reported by Marabini et al. (1994).

In this study treated Duranta erecta $L$ Var. Variegata plants with proline and ascorbic acid increased aniline 4-hydroxylase. Because the activity of aniline 4hydroxylase is induced by an $\mathrm{H}_{2} \mathrm{O}_{2}$ signal generated by various environmental stresses. Aniline 4-hydroxylase are important for the AOS-scavenging system, the investigation on aniline 4-hydroxylase has seldom been reported (Sawada et al. 2006).

Finally, proline and ascorbic acid increased antioxidant enzyme systems to protect plant cells from oxidative enzyme and decreased the toxic ions, DNA breakage $\%$ and increased photosynthetis, growth and survivability (\%).

\section{Conclusions}

Using proline at a rate of $80 \mathrm{ppm}$ or ascorbic acid at a rate of $200 \mathrm{ppm}$ for four months was recommended to get the best growth of Duranta erecta L. var. varigata plants and increased antioxidant enzyme system, which irrigated with seawater concentrations. Plants irrigated with $6000 \mathrm{ppm}$ seawater need to continuesly spraying with proline at $80 \mathrm{ppm}$ to keep alive.

\section{Declarations}

\section{Funding}

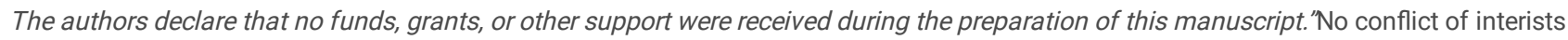

\section{Competing Interests}

All authors certify that they have no affiliations with or involvement in any organization or entity with any financial interest or non-financial interest in the subject matter or materials discussed in this manuscript.

The authors have no relevant financial or non-financial interests to disclose. 
Author Contributions

All authors contributed to the study conception, design, and Material preparation. Data collection was performed by [Magda Ibrahim El-Desouky Hamoud] and [ Sabah El banna]. Data analysis, the first draft of the manuscript were performed by [Magda Ibrahim El-Desouky Hamoud] and [Gehan Gaber Mostafa]. All authors reviewed and commented on previous versions of the manuscript, read and approved the final manuscript

\section{Data Availability}

The datasets estimated during and/or analysed during the current study are available from the corresponding author on reasonable request

\section{References}

Abd El-Samad HM, Shaddad MAK, Barakat N (2010) The role of amino acids in improvement in salt tolerance of crop plants. Journal of Stress Physiology \& Biochemistry, 6 (3), 25-37.

Abdallah MMS, El-Bassiouny HMS (2016). Impact of exogenous proline or tyrosine on growth, some biochemical aspects and yield components of quinoa plant grown in sandy soil. International Journal of Phar. Tec. 9(7):12-23.

Abd-EL-Hady WM, Selim EMM, El-Sayed NI (2019).Influence of humic and ascorbic acids on growth parameters and anthocyanin content of Acalypha wilkesiana irrigated with seawater. Plant Archives, 19(1), 652-664.

Ahmed AH (1996). Physiological studies on tiploun and nitrate accumulation in lettuce plants. J. Agric. Sci., Mansoura Univ., 21: $3971-3994$.

Allu AD, Soja A, Wu A, Szymanski J, Balazadeh S (2014). Salt stress and senescence identification of cross-talk regulatory components.J. Exp. Bot. 65, 39934008.

Alotaibi S, Ali E, Darwesh H, Ahmed AT, Al-Thubaiti E (2019).Effect of Proline on Growth and Nutrient Uptake of Simmondsia chinensis (Link) Schneider under Salinity Stress. Pakistan journal of biological sciences: PJBS, 22(9), 412-418.

Apel K, Hirt H (2004). Reactive oxygen species: metabolism, oxidative stress, and signal transduction. Annu. Rev. Plant Biol. 55, $373-399$.

Awad MM, Abd El-Hameed AM, Shall ZS (2007). Effect of glycine, lysine and nitrogen fertilizer rates on growth, yield and chemical composition of potato. J Agric Sci Mansoura Univ. 32(10):8541-8551.

Bosabalidis AM, Kofidis G (2002). Comparative effects of drought stress on leaf anatomy of two olive cultivars. Plant Science 163(2):375-379.

Butt M, Ayyub CM, Amjad M, Ahmad R (2016). Proline application enhances growth of chilli by improving physiological and biochemical attributes under salt stress. Pak. J. Agri. Sci., 53 (1): 43-49.

Chookhampaeng S (2011). The effect of salt stress on growth, chlorophyll content, proline content and antioxidative enzymes of pepper (Capsicum annuum L.) seedling. Euro. J. Sci., 49(1): 103-109.

Cuin TA, Tian Y, Betts SA, Chalmandrier R, Shabala S (2009). Ionic relations and osmotic adjustment in durum and bread wheat under saline conditions. Funct. Plant Biol., 36: 1110- 1119.

Dolatabadian A, Mohammad S A, Sanavy M, Asilan KS (2010). Effect of ascorbic acid foliar application on yield, yield component and several morphological traits of grain corn under water deficit stress conditions. Notulae Scientia Biologicae, 2: 45-50.

Dore AC (1986). Evaluation of ploidy level in plants of cabbage population of Bruxelles from Brassica oleracea L. pollen origin. Agron., 6 (9): $797-801$.

Ellman M (1959). Aspectrophotometric method for determination of reduced glutathione in tissues. AnalytBiochem, 74, 214-226.

Galal AA, Gad El-Hak SH, Abdel-Ati YY, Moustafa YMM (2000). Response of new tomato hybrids to some antioxidants and early blight. The 2 Scientific Conf. Agric. Sci., Assuit, Egypt, pp: 673-686.

Gomez K. A, Gomez AA (1984). Statistical procedures for agricultural research. John Wiley \& Sons.

Goujon T, Sibout R, Eudes A, MacKay J, Jouanin L (2003). Genes involved in the biosynthesis of lignin precursors in Arabidopsis thaliana. Plant Physiology and Biochemistry, 41(8), 677-687.

Hassanain M A, Osman AR, Sewedan E, Shible Kh (2017). Effect of Natural Substances on Carnation Plants (Dianthus caryophyllus L.) Growing (3), 119-129. Hoque MA, Banu MNA, Nakamura Y, Shimoishi Y, Murata Y (2008). Proline and glycinebetaine enhance antioxidant defense and methylglyoxal detoxification systems and reduce $\mathrm{NaCl}$-induced damage in cultured tobacco cells. Journal of plant physiology, 165(8), 813-824.

https://www.wunderground.com. 
Hussein MM, El-Faham SY, Abd El-Moti EZ, Abou-Baker NH (2017) Jojoba irrigated with diluted seawater as affected by ascorbic acid application. International Journal of Agricultural Research, 12(1), 1-9.

Huxley A, Griffiths M, Levy M (1992). The New Royal Hort. Society Dictionary of Gardening. The Stockton Press, 257 Park Avenue South, New York, N. Y. 10010, USA, (2), 747.

Ibrahim AM, Awad AE, Gendy ASH, Abdelkader MAl. (2019). Effect of proline foliar spray on growth and productivity of sweet basil (Ocimum basilicum, L.) plant under salinity stress conditions. Zagazig Journal of Agricultural Research, 46(6), 1877-1889.

Jaleel CA, Gopi R, Manivannan P, Panneerselvam R (2007). Antioxidative potentials as a protective mechanism in Catharanthus roseus (L.) G. Don. plants under salinity stress. Turkish Journal of Botany, 31(3), 245-251.

Kamran M, Shahbaz M, Ashraf M, Akram NA (2009). Alleviation of drought-induced adverse effects in spring wheat (Triticum aestivum L.) using proline as a pre-sowing seed treatment. Pakistan Journal of Botany 41(2):621-632.

Kato M, Shimizu S (1987). Chlorophyll metabolism in higher plants. VII. Chlorophyll degradation in senescing tobacco leaves; phenolic-dependent peroxidative degradation. Can. J. Bot. 65, 729-735.

Kato R, Gillette JR (1965). Effect of starvation on NADPH-dependent enzymes in liver microsomes of male and female rats.Journal of Pharmacology and Experimental Therapeutics, 150(2), 279-284.

Maia SSS, da Silva RCP, de Oliveira FA, da Silva OMP, da Silva AC, Candido WS (2017). Responses of basil cultivars to irrigation salinity. Rev. Brad. Eng. Agríc. Ambient., 21(1):44-49. Doi.org/10.1590/1807- 1929/agriambi.v21n1 p44-49.

Malkiewicz MK., Smolik B (2019). Alleviative effects of chitosan and ascorbic acid on Petunia atkinsiana D. Don under salinity.Eur. J. Hortic.Sci, 84 (6), 359365.

Marabini L, Radice S, Cipelletti B, Chiesara E (1994). Different amounts of cytochrome P-450 dependent monooxygenases in tulip bulbs, pea seedlings and maize endosperm cells. Plant Science, 99(2), 135-140.

Marcum KB (2006). Use of saline and non - potable water in the turfgrass industry. Constraints and developments. Agric. Water Manag., 80 (1), $132-146$.

Mukherjee SP, Choudhuri MA (1983). Implications of water stress-induced changes in the levels of endogenous ascorbic acid and hydrogen peroxide in Vigna seedlings. Plant Physiol. 58: 166-170.

Nash T (1953). The colorimetric estimation of formaldehyde by means of the Hantzsch reaction. Biochemical Journal, 55(3), 416.

Nishikimi M, Rao NA, Yagi K (1972). The occurrence of superoxide anion in the reaction of reduced phenazinemethosulfate and molecular oxygen.Biochemical and Biophysical Research Communications, 46(2), 849-854.

Omura T, Sato R (1964). The carbon monoxide-binding pigment of liver microsomes I. Evidence for its hemoprotein nature.Journal of Biological Chemistry, 239(7), 2370-2378.

Page AL, Miller RH, Keeney DR (1982). Methods of soil analysis part 2: chemical and microbiological propareties Amer. Soc. Agronomy. Madison, Wisconsin USA., 42(12):1405- 1407.

Pipattanawong N, Tiwong S, Thongyean B, Darak R, Thamin P, Techa W (2008). Improvement of Propagation by Hardwood Cuttings with and without Plastic Pavilions on fig (Ficus carica L.) Kasetsart J (Nat Sci) 42:207-214.

Piper CS (1967). Soil and Plant Analysis 2를 ed., Asia Pub.House. Bombay, India, PP. 85-102.

Pourcel L, Routaboul JM, Cheynier V (2007). Flavonoid oxidation in plants: from biochemical properties to physiological functions. Trends Plant Sci., 12 (1), 29-36.

Rady MM, Taha RS, Mahdi AHA (2016). Prolin enhances growth, productivity and anatomy of two varieties of Lupinus termis L. grown under salt stress, South African Journal of Botany, 102,221-227.

Rahie, A, Smith M, Taton M (1997). The Role of Cytochrome b5in 4a-Methyl-Oxidation and C5 (6) Desaturation of Plant Sterol Precursors. Biochemical and biophysical research communications, 236(2), 434-437.

Ramadan AA, Abd Elhamid E M, Sadak M S (2019). Comparative study for the effect of arginine and sodium nitroprusside on sunflower plants grown under salinity stress conditions. Bulletin of the National Research Centre 43:118-129.

Ramawat KG (2010). Desert plants biology and biotechnology. Kasera, P. K. and Mohammed, S. chapter 14.Springer.

Sadak MS, El-Enany MAM, Bakry B A, Abdallah MMS, El-Bassiouny HMS (2020). Signal Molecules Improving Growth. Yield and Biochemical Aspects of Wheat Cultivars under Water Stress Asian J of Plant Sciences. 19(1):35-53. 
Sairam RK, Srivastava GC, Agarwal S, Meena RC (2005). Differences in antioxidant activity in response to salinity stress in tolerant and susceptible wheat genotypes. Biol Plant.49 (1):85-91.

Sawada H, Shim IS, Usui K (2006). Induction of benzoic acid 2-hydroxylase and salicylic acid biosynthesis-modulation by salt stress in rice seedlings. Plant Science, 171(2), 263-270.

Shalata A, Neumann PM (2001). Exogenous ascorbic acid (vitamin c) increases resistance to salt stress and reduced lipid peroxidation. Journal of Experimental Botany, 52 (364), 2207-2211.

Siddique A B, Islam MR, Hoque MA, Hasan MM, Rahman MT, Uddin MM (2015). Mitigation of salt stress by foliar application of proline in rice. Universal Journal of Agricultural Research, 3(3), 81-88.

Sinha AK (1972). Colorimetric assay of catalase.Analytical Biochemistry, 47(2), 389-394.

Soliman AS, El-feky SA, Darwish E (2015). Alleviation of salt stress on Moringa peregrina using foliar application of nanofertilizers. Journal of Horticulture and Forestry, 7(2), 36-47.

Soltani Y, Saffari VR, Moud AAM (2014). Response of growth, flowering and some biochemical constituents of Calendula officinalis L. to foliar application of salicylic acid, ascorbic acid and thiamine. Ethno- Pharmaceutical products, 1(1):37-44.

Soussi M, Santamoria M, Ocana A, Liuch C (2001). Effect of salinity on protein and lipopolysaccharide pattern in salt tolerant strain of Mesorhizobium ciceri. J Appl Microbiol.;90:476-481.

Tappel AL, Zalkin H (1959). Inhibition of lipide peroxidation in mitochondria by vitamin E. Archives of Biochemistry and Biophysics, 80(2), $333-336$.

Tester M, Davenport R (2003). Na+ tolerance and Na+ transport in higher plants. Annals of Botany 91: 503-527.

Venkatesan A, Chellappan KP (1998). Accumulation of proline and glycine betaine in Ipomoea peacaprae induced by NaCl.Biol Plant. 41:271-276.

Wu B, Ootani A, Iwakiri R, Sakata Y, Fujise T, Amemori S, Fujimoto K (2006). T cell deficiency leads to liver carcinogenesis in Azoxymethane-treated rats. Experimental Biology and Medicine, 231(1), 91-98.

Wu GQ, Feng RJ, Li SJ, Du YY (2017). Exogenous application of proline alleviates salt-induced toxicity in sainfoin seedlings. J Anim Plant Sci, $27,246-251$.

Yadawa UL (1986). Arapid and non-destructive method to determined chlorophyll in intact leaves. Hort. Sci., 21(6):1449-1450.

Zhao H, Liang H, Chu Y, Sun C, Wei N, Yang M, Zheng C (2019). Effects of salt stress on chlorophyll fluorescence and the antioxidant system in Ginkgo biloba L. seedlings. HortScience, 54(12), 2125-2133. 